\title{
Cross Talk Experiment with Two-element CdTe Detector and Collimator for BNCT-SPECT
}

\author{
Masanobu Manabe*, Ryosuke Ohya, Nobuhide Saraue, Fuminobu Sato, Isao Murata \\ Osaka University, Osaka, Japan
}

\section{Original Research}

Received May 12, 2016

Revision August 24, 2016

Accepted September 12, 2016

Corresponding author:

Masanobu Manabe

Osaka University, 1-1 Yamada, Suita,

Osaka 565-0871, Japan

Tel: +81-6-6879-7894

Fax: +81-6-6879-7899

E-mail:manabe@qr.see.eng.osaka-u.ac.jp

This is an Open-Access article distributed under the terms of the Creative Commons Attribution NonCommercial License (http://creativecommons.org/ licenses/by-nc/4.0) which permits unrestricted noncommercial use, distribution, and reproduction in any medium, provided the original work is properly cited.

Copyright $\odot$ 2016 The Korean Association for Radiation Protection

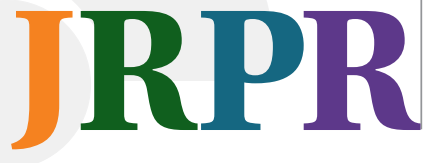

Background: Boron Neutron Capture Therapy (BNCT) is a new radiation therapy. In BNCT, there exists some very critical problems that should be solved. One of the severest problems is that the treatment effect cannot be known during BNCT in real time. We are now developing a SPECT (single photon emission computed tomography) system (BNCT-SPECT), with a cadmium telluride (CdTe) semiconductor detector. BNCT-SPECT can obtain the BNCT treatment effect by measuring $478 \mathrm{keV}$ gamma-rays emitted from the excited state of ${ }^{7} \mathrm{Li}$ nucleus created by ${ }^{10} \mathrm{~B}(\mathrm{n}, \alpha){ }^{7} \mathrm{Li}$ reaction. In the previous studies, we investigated the feasibility of the BNCTSPECT system. As a result, the $S / N$ ratio did not meet the criterion of $S / N>1$ because deterioration of the $\mathrm{S} / \mathrm{N}$ ratio occurred caused by the influence of Compton scattering especially due to capture gamma-rays of hydrogen.

Materials and Methods: We thus produced an arrayed detector with two CdTe crystals to test cross talk phenomenon and to examine an anti-coincidence detection possibility. For more precise analysis for the anti-coincidence detection, we designed and made a collimator having a similar performance to the real BNCT-SPECT.

Results and Discussion: We carried out experiments with the collimator to examine the effect of cross talk of scattering gamma-rays between CdTe elements more practically. As a result of measurement the coincidence events were successfully extracted.

Conclusion: We are now planning to carry out evaluation of coincidence rate from the measurement and comparison of it with the numerical calculations.

Keywords: BNCT, SPECT, CdTe detector, Gamma-ray, Coincidence detection

\section{Introduction}

Boron Neutron Capture Therapy (BNCT) is a new radiation therapy which attracts the whole world's attention. This therapy can kill tumor cells by alpha particles $(\alpha)$ and lithium nuclei $\left({ }^{7} \mathrm{Li}\right)$ emitted by the reaction of thermal neutron or epithermal neutron with boron $\left({ }^{10} \mathrm{~B}\right)$. In BNCT, there exists some very critical problems that should be solved. One is that the treatment effect cannot be known during BNCT in real time. Many researchers [1-4] have proposed imaging systems to see the treatment effect in real time in order to solve this problem. We are now developing a SPECT (single photon emission computed tomography) system to measure it, named BNCT-SPECT, with a cadmium telluride (CdTe) semiconductor detector [5].

As indicated in Figure 1, BNCT-SPECT can obtain BNCT treatment effect by measuring $478 \mathrm{keV}$ gamma-rays emitted from the excited state of ${ }^{7} \mathrm{Li}$ nucleus created by ${ }^{10} \mathrm{~B}(\mathrm{n}, \mathrm{\alpha}){ }^{7} \mathrm{Li}$ reaction. 


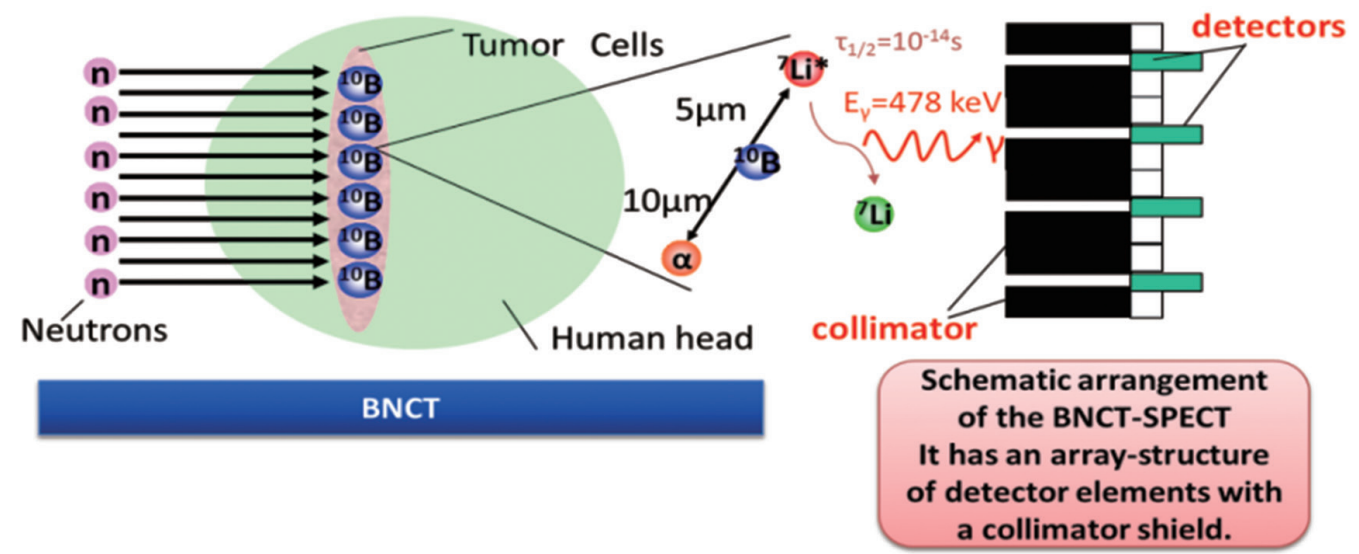

Fig. 1. The principle of BNCT-SPECT.

Precisely, ${ }^{10} \mathrm{~B}(\mathrm{n}, \mathrm{\alpha})^{7} \mathrm{Li}$ reaction utilized in BNCT is practically expressed by the next two nuclear reactions:

$$
\begin{aligned}
{ }^{10} \mathrm{~B}+\mathrm{n} \rightarrow & { }^{7} \mathrm{Li}+\alpha+2.79 \mathrm{MeV}(6.1 \%) \\
& { }^{7} \mathrm{Li}^{*}+\alpha+2.31 \mathrm{MeV}(93.9 \%) \\
\rightarrow & { }^{7} \mathrm{Li}+\gamma(478 \mathrm{keV})
\end{aligned}
$$

$94 \%$ of ${ }^{7} \mathrm{Li}$ is in a first excited state, i.e., ${ }^{7} \mathrm{Li}^{*} .{ }^{7} \mathrm{Li}^{*}$ decays in its half-life of 10-14 seconds to emit a $478 \mathrm{keV}$ gamma-ray via transition from the first excited state to the ground state.

If the intensity distribution of $478 \mathrm{keV}$ gamma-rays could be measured three-dimensionally, we could obtain the three-dimensional distribution of ${ }^{10} \mathrm{~B}(\mathrm{n}, \alpha){ }^{7} \mathrm{Li}$ reaction rate in the tumor. The result of the measurement can then be regarded as the treatment effect of BNCT. Emitted $478 \mathrm{keV}$ gamma-rays are collimated by the collimator and measured by a lot of measuring devices in the array detector in order to make an image of gamma-ray intensity. The BNCT treatment effect (local tumor dose) can be estimated from the obtained three dimensional gamma-ray image.

BNCT-SPECT should be so designed that $478 \mathrm{keV}$ gammarays have to be measured in a very high background field. The measurement thus has to be carried out carefully from a viewpoint of actual medical situation and site [6].

Considering the above condition, we selected a CdTe detector so as to detect gamma-rays of $478 \mathrm{keV}$. The reason is that it has a good energy resolution and it can be used without casing and cooling. We have already made clear an optimum size of the CdTe element, the performance test of which was carried out so far [7-9]. According to the previous studies, the estimated count rate of $478 \mathrm{keV}$ gamma-rays was sufficiently large being more than the target value of over

\section{1,000 counts/hour.}

However, the signal to noise $(\mathrm{S} / \mathrm{N})$ ratio did not meet the criterion of $\mathrm{S} / \mathrm{N}>1$. We then tried to improve the $\mathrm{S} / \mathrm{N}$ ratio, because we confirmed that deterioration of the $\mathrm{S} / \mathrm{N}$ ratio was caused by the influence of Compton scattering especially due to capture gamma-rays of hydrogen [10].

We thus produced an arrayed detector with two CdTe crystals to experimentally make clear cross talk phenomenon by Compton scattering gamma-rays and finally to examine an anti-coincidence detection possibility to improve the $\mathrm{S} / \mathrm{N}$ ratio. We carried out anti-coincidence measurement with a standard gamma-ray source, ${ }^{137} \mathrm{Cs}$, and confirmed possibility of reduction of noises formed by Compton scattering of incident gamma-rays [11].

In this study, for more precise analysis for the anti-coincidence detection, we carried out experiments with a collimator having a similar performance to the real BNCT-SPECT.

\section{Materials and Methods}

\section{Two-element CdTe detector}

Figure 2 shows a set of two-element CdTe detector. We set two CdTe elements $(1.5 \times 2 \times 30 \mathrm{~mm}$ and $1.5 \times 2 \times 35 \mathrm{~mm})$ in one board. One crystal is the largest size we have and the other one shows the best performance among our CdTe crystals. As shown in Figure 2B, the CdTe elements are well shielded from light by a special case having windows of gamma-ray entrance.

In this experiment, we used a set of two-element CdTe detector, not an arrayed detector detailed in Ref. [11]. It means the set of two-element CdTe detector has a gap between the two. By using the set of two-element, the coincidence rate by 


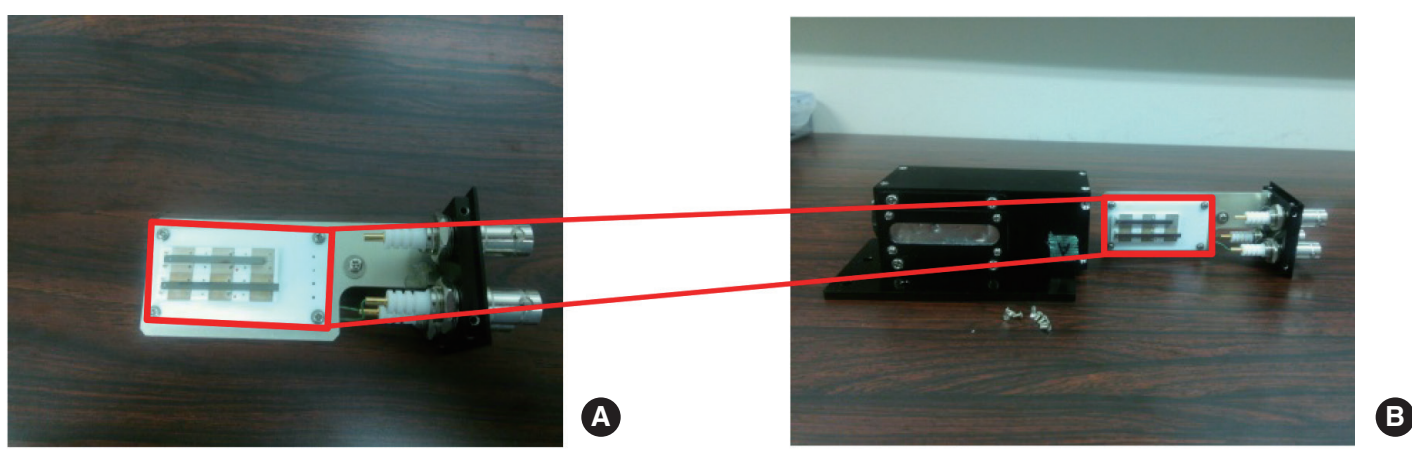

Fig. 2. Photos of a set of two-element CdTe detector; (A) Two-element CdTe detector and (B) Set of two-element CdTe detector.

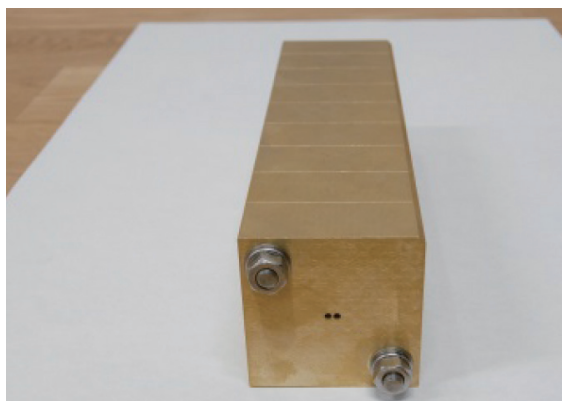

A

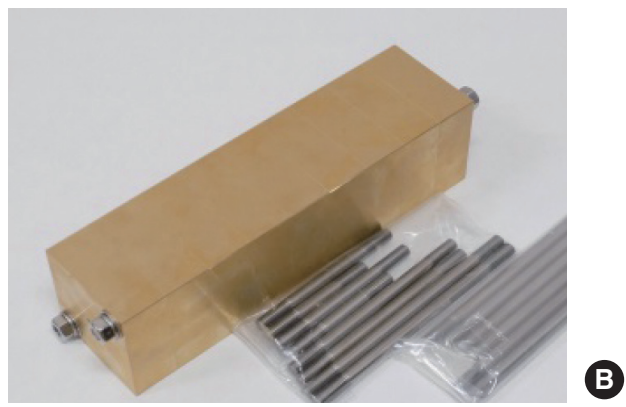

Fig. 3. Photos of designed collimator; (A) Front view of the collimator and (B) Oblique view of the collimator.

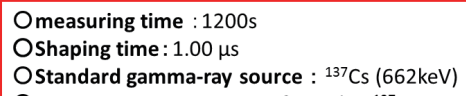

ODetector position: $7.2 \mathrm{~cm}$ from the ${ }^{137} \mathrm{Cs}$ gamma-ray source

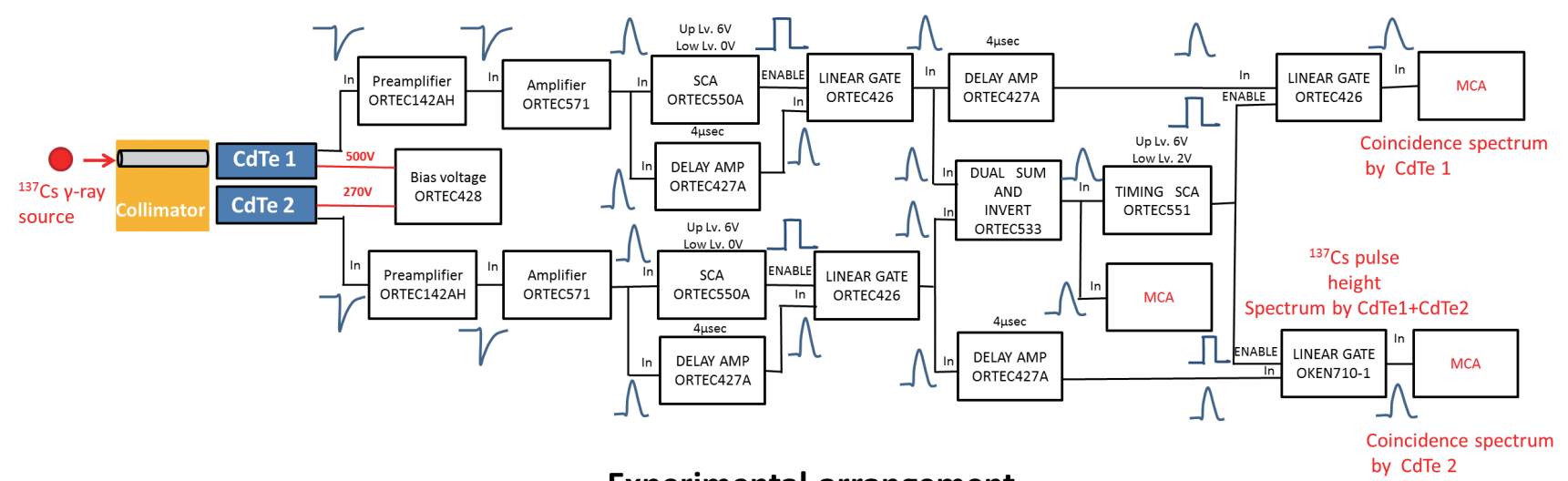

\section{Experimental arrangement}

Fig. 4. Electronic circuits of coincidence measurement.

cross talks could be more accurately examined because the elements are apart from each other.

\section{Collimator}

We designed and produced a collimator which has a similar performance of the one originally designed for the array type CdTe detector [10]. Figure 3 shows the collimator. The collimator is made from brass. The size of the collimator is $5 \times 5 \times 18 \mathrm{~cm}$ and it has two collimator holes, each of which has a diameter of $2 \mathrm{~mm}$ and is positioned with a pitch of 2.5 $\mathrm{mm}$. Also, the collimator length can be varied with intervals of $2 \mathrm{~cm}$ by stacking $2 \mathrm{~cm}$ thick collimator ports with metal rods in Figure 3B prepared corresponding to the total collimator length. 

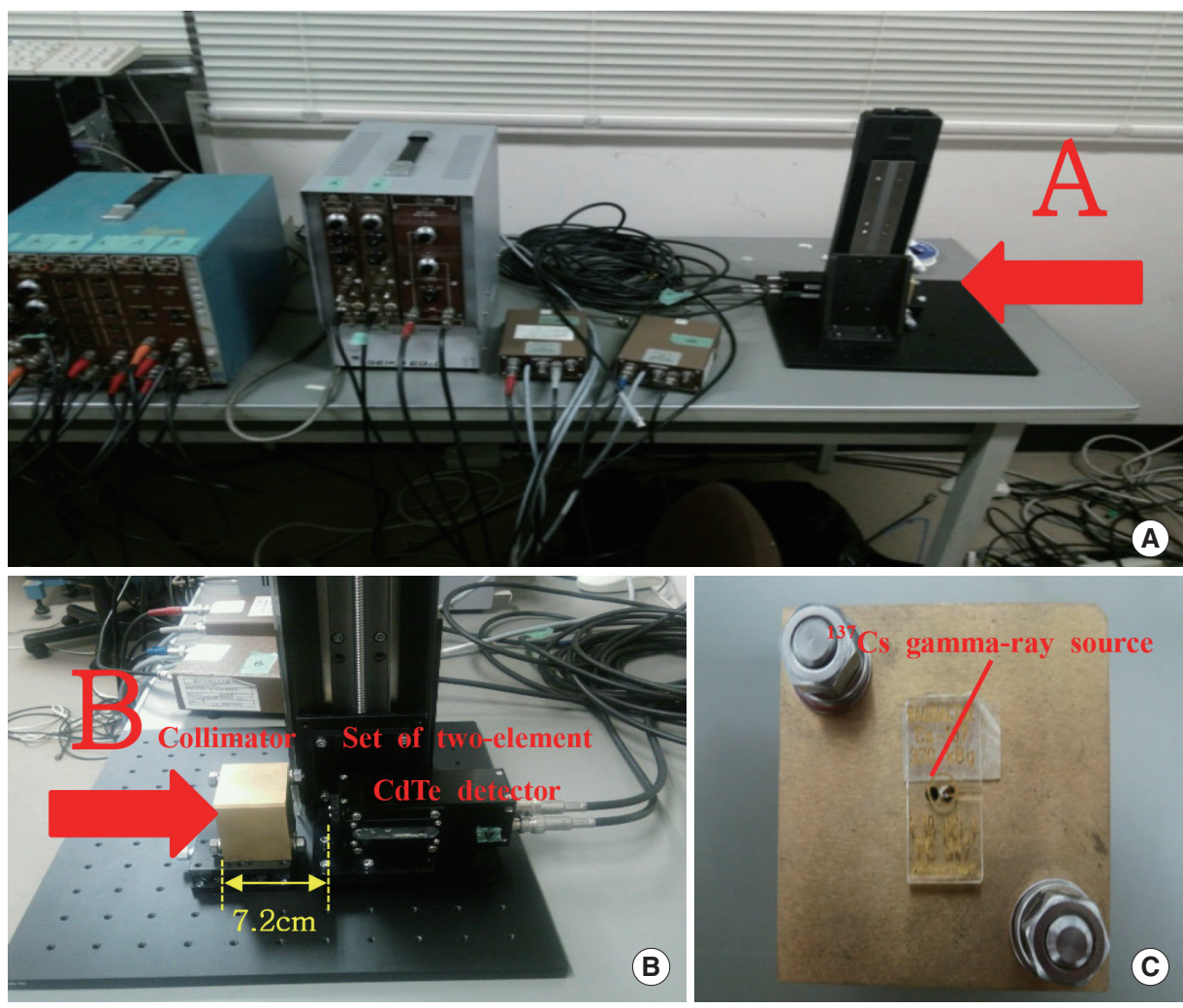

Fig. 5. Photos of experimental arrangement; (A) Electronic circuits of coincidence measurement, (B) The two-element CdTe detector and the collimator (from direction $A$ in $(A)$ ), and (C) Enlarged view of the collimator (from direction $B$ in $(B))$.

\section{Experimental system}

We examined basic property of coincidence events between two CdTe elements by measuring coincidence signals of the two to confirm improvement of the $\mathrm{S} / \mathrm{N}$ ratio. Figures 4 and 5 show the electronic circuits and experimental arrangement used in this study. By this circuit three spectra can be obtained, coincidence spectrum by CdTe 1 positioned behind the collimator hole, coincidence spectrum by CdTe 2 and the sum spectrum of both coincident signals. At first, a ${ }^{137} \mathrm{Cs}$ gamma-ray source of $410 \mathrm{kBq}(23 \times 10 \times 2 \mathrm{~mm})$ was set on the collimator hole so that gamma-rays pass through the collimator and reach CdTe 1. The length of the collimator was decided to be $4 \mathrm{~cm}$ to prevent source gamma-rays entering directly into CdTe 2 . Next, power supply voltages of $\mathrm{CdTe} 1$ and CdTe 2 were set to be $500 \mathrm{~V}$ and $270 \mathrm{~V}$, respectively because it is necessary to adjust each voltage due to individual crystal property.

\section{Results and Discussion}

Figure 6 shows results of a measurement by the set of twoelement CdTe detector. In Figure 6A and B, coincident spec- tra are described. In Figure 6A, is shown a coincident spectrum of CdTe 1 positioned just behind the collimator hole, meaning that source gamma-rays are incident directly to the detector. As in the figure there are two peaks, in which a higher energy one corresponds to the photo peak and a lower energy one is a Compton electron energy distribution. The reason why this kind of peak is obtained is that the detector is very long though the entrance window of the detector is very small. In Figure 6B, the spectrum of the CdTe 2 placed just adjacent to CdTe 1 is shown. In this case, the detector is not positioned behind the collimator hole. Gamma-rays are thus entering only from CdTe 1, not from the source.

As a result, as shown in Figure 6B, just a low energy signal made of Compton electron of Compton scattering gammarays produced in CdTe 1 is seen. To verify the measurement, the summation of the both signals of CdTe 1 and CdTe 2 was measured. Figure 6C shows the summation spectrum of both coincident signals. In the figure one can confirm that a clear photo-peak of the ${ }^{137} \mathrm{Cs}$ certainly exists. This means the present measurement could successfully extract coincident signals of the two detectors. 


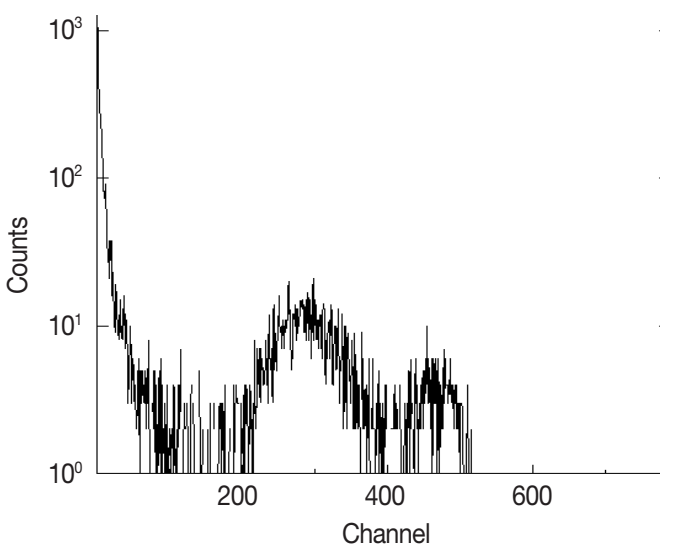

(A)

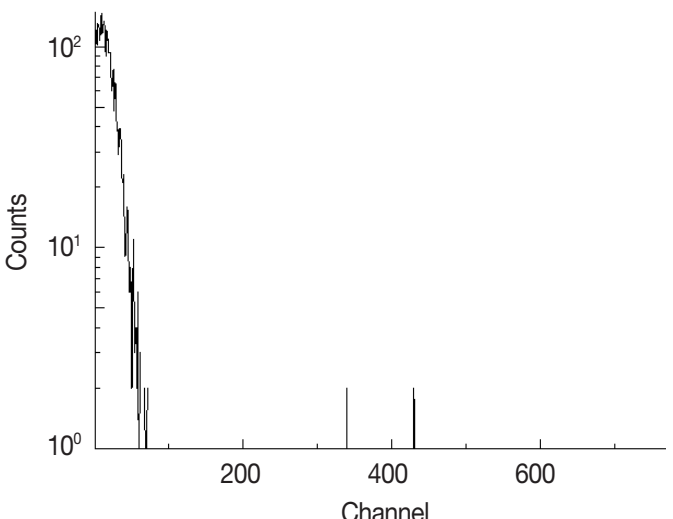

B

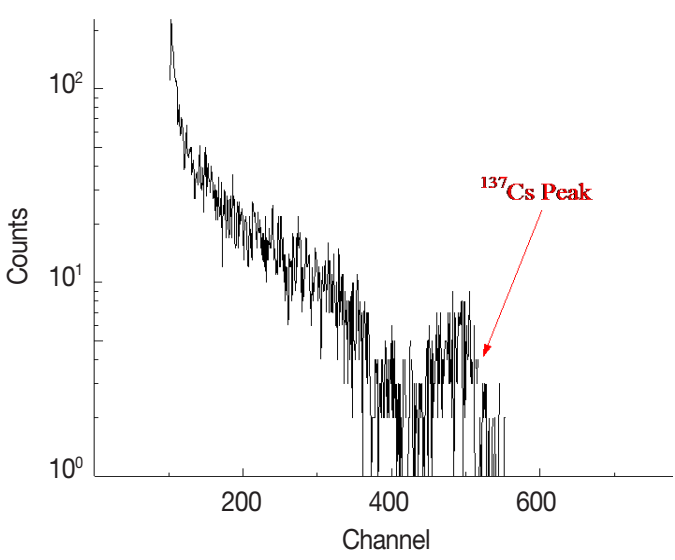

Fig. 6. Measured pulse height spectra (PHS) of ${ }^{137} \mathrm{Cs}$ and coincidence events; (A) The CdTe1 PHS with the collimator, (B) The CdTe 2PHS without the collimator, and (C) The sum PHS of the CdTe1 and the CdTe2.

\section{Conclusion}

We carried out coincident spectrum measurement for BNCT-SPECT development with a set of two-element CdTe detector with a collimator and standard gamma-ray source of ${ }^{137}$ Cs.

As a result of measurement the coincidence events were successfully extracted. This result is very valuable for designing of BNCT-SPECT. We are now planning to carry out evaluation of coincidence rate from the measurement and comparison of it with the numerical calculations.

\section{References}

1. Kobayashi T, Sakurai Y, Ishikawa M. Anoninvasive dose estimation system for clinical BNCT based on PG-SPECT-Conceptual study and fundamental experiments using HPGe and CdTe semiconductor detectors. Med. Phys. 2000; 27(9): 2124-2132.

2. Minsky DM, Valda AA, Kreiner AJ, Green S, Wojnecki C, Ghani Z. Experimental feasibility studies on a SPECT tomograph for BNCT dosimetry. Appl. Radiat. Isot. 2009;67:S179-S182.

3. Rosenschöld P. M, MinarikD, Östlund C, Ljungberg M, Ceberg C. Prompt gamma tomography during BNCT -A feasibility study. J. Instrum. 2006;1:wP05003.

4. Katabuchi T, Hales B, Hayashizaki N, Igashira M, Khan Z, Kobayashi T, Matsuhashi T, MiyazakiK, Ogawa K, Terada K. Feasibility study on pinhole camera system for online dosimetry in boron neutron capture therapy. Appl. Radiat. Isot. 2014;88:139142.

5. Murata I, Mukai T, Ito M, Miyamaru H, Yoshida S. Feasibility study on BNCT-SPECT using a CdTe detector. Progress in Nucl. Sci. Technol. 2011;1:267-270.

6. Manabe M, Nakamura S, Murata I. Present status of BNCTSPECT development with CdTe detector. Proc. of the 2013 Symposium on Nuclear Data, Nov.14-15,Fukui, Japan JAEA-Conference 2014. 2015;002:198-203.

7. Mukai T, Murata I, Ito M, Miyamaru H. Development of CdTe detector for BNCT-SPECT. Proc. of the 2009 Annual Symposium on Nucl. Data, Nov. 26-27, 2009, RICOTTI, Tokai-mura, Ibarakiken, Japan. JAEA-Conference. 2010;005:75-80.

8. Murata I, Mukai T, Nakamura S, Miyamaru H, Kato I. Development of a thick CdTe detector for BNCT-SPECT. Appl. Radiat. Isot. 2011;69:1706-1709.

9. Nakamura S, Mukai T, Manabe M, Murata I. Characterization test of CdTe detector element designed and developed for BNCT-SPECT. Proc. of the 2011 Annual Symposium on Nucl. Data, Nov. 16-17, 2011, RICOTTI, Tokai-mura, Ibaraki-ken, Japan. JAEA-Conference. 2012;001:165-170.

10. Manabe M, Nakamura S, Murata I. Study on measuring device arrangement of array-type CdTe Detector for BNCT-SPECT. Rep. Pract. Oncol. Radiother. 2016;21(2):102-107.

11. Manabe M, Sato F, Murata I. Basic detection property of an array-type CdTe detector for BNCT-SPECT -Measurement and analysis of anti-coincidence events-. Appl. Radiat. Isot. 2016;118: 389-394. 